\title{
Rupture mechanics of vimentin intermediate filament
}

\section{tetramers}

Markus J. Buehler ${ }^{1, *}$

${ }^{1}$ Esther and Harold E. Edgerton Assistant Professor, Laboratory for Atomistic and Molecular Mechanics, Department of Civil and Environmental Engineering, Massachusetts Institute of Technology, 77 Massachusetts Ave. Room 1-272, Cambridge, MA, 02139, USA

*Corresponding author, E-mail: mbuehler@MIT.EDU

ABSTRACT: Together with the globular proteins microtubules and microfilaments, intermediate filaments are one of the three major components of the cytoskeleton in eukaryotic cells. It consists of a dimeric coiled-coil building block, assembled in a very precise, hierarchical fashion into tetramers, forming filaments with characteristic dimensions on the order of several micrometers. Here we focus on the theoretical analysis of the deformation mechanics of vimentin intermediate filaments, a type of intermediate filament expressed in leukocytes, blood vessel endothelial cells, some epithelial cells, and mesenchymal cells such as fibroblasts. The main contribution of this paper is the study of the rupture mechanics of intermediate filament tetramers, representing an assembly of two dimers, by utilizing a statistical Bell model adapted to describe the rupture dynamics of intermediate filaments. Possible deformation mechanisms, including interdimer sliding and uncoiling of the dimer, are illustrated in light of the interdimer adhesion and dimer stability. The analysis reveals that the 
dominating deformation mechanism depends critically on the interdimer adhesion, solvent condition and deformation rate.

Keywords: Intermediate filaments, cell mechanics, failure, atomistic, molecular, mechanical properties, hierarchical structure

Under submission to: Journal of Engineering Mechanics (ASCE) 


\section{Introduction}

The intermediate filament (IF) is an important component of the cell's cytoskeleton, next to microtubulus and actin filaments (Wang and Stamenovic 2002). The cytoskeleton plays a critical role in determining the shape and the mechanical properties of the cell, and is vital for many additional functions including cell motility as well as cell division (Wang and Stamenovic 2002; Helfand, Chang et al. 2004; Mucke, Kreplak et al. 2004). The vimentin intermediate filament is a particular type, which is typically expressed in leukocytes, blood vessel endothelial cells, some epithelial cells, and mesenchymal cells such as fibroblasts (Alberts, Johnson et al. 2002). Vimentin and all other IF systems are marvelous assembly systems that form highly controlled, hierarchical structures arranged in a precise assembly pattern (Smith, Hempstead et al. 2003; Kreplak, Aebi et al. 2004).

Like many other biological materials, IFs are hierarchical structures with highly specific features at nanoscale (Smith, Hempstead et al. 2003; Kreplak, Aebi et al. 2004). Vimentin consists of a dimeric coiled-coil (CC) building block, assembled in a very precise, hierarchical fashion into tetramers, eventually forming filaments of approximately $10 \mathrm{~nm}$ diameter (the tetramer structure can alternatively also be considered a quaternary structure). Figure 1 depicts the molecular assembly pattern, from the cellular cytoskeleton network reaching down to the molecular protein structure. Figure 2 depicts details of the molecular building block of vimentin IF, the CC dimer, illustrating the four domains of the CC structure as well as the head and tail domain.

Since the CC structure is essential for the vimentin IF network, some key features of the $\mathrm{CC}$ structure are briefly reviewed in this section. Each $\mathrm{CC}$ consists of a single alpha 
helical $(\mathrm{AH})$ protein. An $\mathrm{AH}$ is generated when a single polypeptide chain twists around on itself, stabilized by hydrogen bonds (H-bond or HBs) made between every fourth residue, linking the $\mathrm{O}$ backbone atom of peptide $i$ to the $\mathrm{N}$ backbone atom of peptide $i+4$ in the polypeptide chain. Consequently, at each convolution, $3.5 \mathrm{H}$-bonds are found in a parallel arrangement that stabilize the helical configuration (Alberts, Johnson et al. 2002). A particularly stable molecular configuration of $\mathrm{AH}$ based protein structures are alpha-helical coiled-coils (CCs), which appear in approximately 10\% of all proteins (Rose and Meier 2004). The CC consist of an assembly of two or more AHs in which the primary structure reveals a pronounced seven residue periodicity called a heptad repeat. Within this repeat, first and fourth positions are preferably occupied with nonpolar residues (Strelkov, Herrmann et al. 2003; Maccallum, Moghaddam et al. 2007) such as Leu, Ala, Val or Ile. The hydrophobic residues, concentrated on one side of the helix, are the reason why the proteins assemble into the CC structure: In order to avoid contact with surrounding water molecules, AHs assemble into CCs by wrapping around each other, clustering the hydrophobic side chains inside (Alberts, Johnson et al. 2002). In addition, interhelical and intrahelical salt bridges contribute to the CC's thermodynamic stability (Burkhard, Kammerer et al. 2000).

Vimentin IFs have a variety of functions on various hierarchical levels. On the cellular scale, vimentin networks act primarily as the 'security belts' of the cell (Burkhard, Kammerer et al. 2000). Due to their architecture, vimentin networks are very flexible and appear to be very soft at small deformation and at small pulling rates, leading to 'invisibility' and non-resistance during cell movement. Contrarily, a very stiff behavior is observed at large deformation and high deformation rates, ensuring their function on 
the cellular as well as on the tissue level (Mucke, Kreplak et al. 2004). In particular, IF networks have been shown to provide resistance to large deformation of cells (Herrmann and Aebi 2004; Mucke, Kreplak et al. 2004; Strelkov, Schumacher et al. 2004). It has been hypothesized that IFs are critical to provide strength to the cell under large deformation, and to absorb large amounts of energy upon a certain load by unfolding (Fudge, Gardner et al. 2003; Fudge and Gosline 2004). IFs do not participate in any cell movement (Alberts, Johnson et al. 2002).

Recently, additional functions were found on the sub-network filament level, which control the location, shape and stability of cell organelles (e.g. mitochondria or golgi) and their function as well as the protein targeting process (Toivola, Tao et al. 2005). And yet other functions exist on the molecular level, consisting of different regulation mechanisms such as cell signaling (e.g. transcriptional effects, mechanotransduction), or associated protein organization (e.g. plectin or chaperones) (Bruck, Evans et al. 2002; Toivola, Tao et al. 2005).

Overall, vimentin IFs are primarily associated with mechanotransduction and with carrying passive loads applied to cells. Since IF filaments span from the cell's nucleus to the cell membrane and therefore interact with IF networks of other cells suggests that IFs play an important role in transmitting mechanical signals from the plasma membrane to the nucleus, where a specific response can be triggered by mechanical stimulation (Moir and Spann 2001; Wilson, Zastrow et al. 2001). It is noted that in contrast to MTs and MFs, IFs do not participate in the dynamic functions of the cytoskeleton. Further, 
they do not support active transport of motor proteins such as myosin and kinesin, due to the missing polarity in the protein structure (Strelkov, Herrmann et al. 2003).

These points underline the static-mechanical role of IFs. Finally, the mechanical role of intermediate filaments is particularly evident in diseases in which the loss of mechanical function and integrity of various tissues is associated with intermediate-filament-protein mutations (Omary, Coulombe et al. 2004; Kiss, Karsai et al. 2006). It was shown that mutations in keratin IFs reduce the ability of these IF networks to bundle and to resist large deformation (Wang and Stamenovic 2002). Furthermore, it has been suggested that point mutations lead to the aggregation of the cytoskeleton and extensive cell fragility in epidermis, heart and skeletal muscle after they are exposed to mechanical strain (Schietke, Brohl et al. 2006). These examples clearly illustrate the significance of the mechanical properties of IF proteins for biological processes.

Table 1 provides an overview over various biological terms used in this paper, explaining terms including cytoskeleton, intermediate filaments, assembly, residue and others.

\section{Review of previous experimental and theoretical work}

In recent years, a variety of $\mathrm{AH}$ structures and $\mathrm{CC}$ s have been studied in experiment (Kreplak, Bar et al. 2005; Kreplak and Fudge 2007) as well as in simulation (Rohs, Etchebest et al. 1999; Mitsui, Nakajima et al. 2000; Cieplak, Hoang et al. 2002;

Schwaiger, Sattler et al. 2002; Akkermans and Warren 2004; Ortiz, Nielsen et al. 2005; Bornschlogl and Rief 2006; Hanke and Kreuzer 2006; Paramore and Voth 2006; Root, Yadavalli et al. 2006; Wolgemuth and Sun 2006; Brockwell 2007; Day and Daggett 
2007; Finke, Jennings et al. 2007; Forman and Clarke 2007; Randles, Rounsevell et al. 2007; Ackbarow and Buehler, 2007).

AFM experiments on single molecules of double-headed myosin, single-headed myosin as well as CC tail fragments were reported in (Cieplak, Hoang et al. 2002; Akkermans and Warren 2004) (these protein structures feature a similar molecular architecture with a dominance of CC domains., albeit their overall structure and biological role is different). It was found that the transition to unfolding of the protein structure (in the following referred to as 'angular point' (AP) or 'unfolding force') begins at strains of about $20 \%$ of stretched protein length. Furthermore, it was shown that myosin is a very elastic protein, with almost no hysteresis at small pulling rates. Even after the $\mathrm{CC}$ structure has been unfolded completely under application of mechanical forces, it was shown that it refolds again to its initial configuration in less than one second (Akkermans and Warren 2004).

Some of the characteristics of myosin deformation during tensile tests have also been observed in MD simulations (Cieplak, Hoang et al. 2002). MD simulations have been carried out also on single AHs (Rohs, Etchebest et al. 1999; Bornschlogl and Rief 2006; Ackbarow, Chen et al. 2007). By applying tensile loads, unfolding of the helical structure has been observed after a short steep increase in force. Thereby, the 20 residues long helix in (Rohs, Etchebest et al. 1999) started to unfold at both ends simultaneously, in contrast to protein structure modeled in (Bornschlogl and Rief 2006), which unfolded systematically from the side where force was applied. Furthermore, a multi-scale model for human hair that mainly consists of keratin IF CCs was developed in (Akkermans and Warren 2004). 
However, aside from these studies, to the best of our knowledge no MD results related to the nanomechanical behavior specifically of IFs, considering the particular features of the molecular assembly, have been published thus far. In particular, no systematic studies on the difference of mechanical properties between the behavior of CCs and assemblies of CCs have been reported, despite the fact that this is crucial for the understanding of the biological-mechanical role of IFs inside the cell.

The lack of models and understanding motivates the analysis presented here, to elucidate of the mechanical behavior of two dimers. This extends earlier work that was purely focused on the mechanics of individual dimers (Ackbarow and Buehler, 2007). Details of this earlier work will be reviewed in Section 2.2.

\section{Structure of vimentin tetramers}

In this article, the focus is on the deformation mechanics of intermediate filament tetramers, and the illustration of possible deformation mechanisms that mediate macroscopic tensile deformation of filaments (for the geometry of filaments, see Figure 1). The central point of the analysis is an estimate of the interaction forces between two $\mathrm{CC}$ dimers to better understand the dominant deformation mechanism under tensile loading of IF filaments. The theoretical study reported here is based on earlier MD simulation results (Ackbarow and Buehler, 2007). To facilitate the analysis, entropic elastic effects are not included. The study is focused purely on strength properties.

A schematic of the vimentin dimer structure is shown in Figure 2. The rod-like structure is 310 residues long and consists of four CCs (1A, 1B, 2A, 2B), divided by several 
linkers (Janmey, Euteneuer et al. 1991; Strelkov, Herrmann et al. 2002; Strelkov, Herrmann et al. 2003; Mucke, Wedig et al. 2004). The structure of parts of the dimer structure has been determined by $\mathrm{x}$-ray diffraction experiments and has been deposited in the Protein Data Bank (PDB) (Janmey, Euteneuer et al. 1991; Strelkov, Herrmann et al. 2002; Strelkov, Herrmann et al. 2003; Mucke, Wedig et al. 2004). PDB structures provided the basis for the MD simulations reported in reference (Ackbarow and Buehler, 2007) (PDB ID 1GK6), forming the basis of the present study.

Figure 3 depicts how interdimer adhesion is facilitated. Extensive experimental analyses of the assembly process revealed that the adhesion between two CCs is dominated by the interaction of the head domain with the other dimer (the head domain is indicated with the red color) (Strelkov, Herrmann et al. 2003; Kreplak, Aebi et al. 2004). The head domain is primarily positively charged at $\mathrm{pH} 7$ (due to approximately $15 \%$ Arg residues), and folds back into the $\mathrm{CC} 1 \mathrm{~A}$ domain, primarily negatively charged at $\mathrm{pH} 7$, and also connects to the $2 \mathrm{~B}$ domain of the other dimer, which is also negatively charged (the $1 \mathrm{~A}$ and 2B domains contain approximately $11 \%$ Glu and Asp residues). Details of the interdimer bonding are schematically shown in the blow-up (lower part of Figure 3).

We note that it is believed that the two CCs itself do not interact via chemical bonds (covalent or weak interactions), which has been confirmed by analyses of the intermolecular distance between the two AHs (Kreplak, Aebi et al. 2004).

Figure 4 illustrates how lateral tensile load, applied at the level of a tetramer, is transmitted via interdimer shear traction. 


\section{Theoretical model}

The description of the rupture mechanics of protein structures (bonded by weak noncovalent interactions) requires incorporation of statistical models that explicitly consider the energy landscape of the interatomic or intermolecular bonding.

We first review the general theoretical approach of treating the strength of proteins and protein assemblies in Section 2.1, and then focus on the nanomechanical behavior of an IF dimer in Section 2.2.

\section{Extended Bell Theory}

Mechanical loading of protein structures can result in severe changes in the protein structure, leading to unfolding of the protein. Typically, a variety of unfolding processes exist for a given protein structure, each of which has a specific reaction pathway and an associated energy barrier, mostly related to specific bond breaking mechanisms and rearrangements in the protein structure. These unfolding modes can be understood as the interplay between different processes with different activation barriers operating at different activation distances. Several theories exist that describe competing processes due to mechanically induced instabilities of protein structures. These concepts stem primarily from the field of physical chemistry (Evans and Ritchie 1997; Gilli, Bertolasi et al. 2004; Dudko, Hummer et al. 2006; Wiita, Ainavarapu et al. 2006), and some of them are derived from a theory originally postulated by Bell in 1978 (Bell 1978). Bell's model represents one of the simplest models to describe the unfolding mechanism in protein structures. Whereas it can not be used to describe some of the more complex protein 
unfolding mechanisms, it provides a reasonable model for simpler protein domains such as the alpha-helical motif studied here.

In Bell's theory, the off rate $\chi$ is the product of a natural vibration frequency, $\omega_{0}$, of the bond in vacuum and the quasi-equilibrium likelihood of reaching the transition state with an energy barrier $E_{b}$ that is reduced by mechanical energy $f \cdot x_{b}$, where $f$ is the applied force, $x_{b}$ is the distance between the equilibrated state (minimum of the well) and the transition state (see Figure 5 for a schematic).

Consequently, the off rate (also known from chemical reaction kinetics) is given by $\chi=\omega_{0} \cdot \exp \left(-\frac{\left(E_{b}-f \cdot x_{b}\right)}{k_{b} \cdot T}\right)$.

The off rate describes how often a bond is broken per unit time and equals to the reciprocal of the lifetime of a bond. The natural vibration frequency of a bond is $\omega_{0} \approx 1 \times 10^{13} \mathrm{~s}^{-1}$ (Bell 1978), $k_{b}$ is Boltzmann constant, $T$ is the temperature, and $x_{b}$ is the distance of the transition point (see also Figure 5).

However, equation (1) does not describe the dependence of the pulling speed (the controlled parameter in experiment and in steered MD simulation) at which a bond breaks due to the applied pulling force. Instead, it only provides an estimate of the time scale at which the bond will be broken. In order to overcome this limitation, we modify equation (1) based on the following idea: The speed $v$ at which a bond is broken equals to the distance that needs to be overcome in order to break the bond $\left(x_{b}\right)$ divided by the time for the bond breaking (the parameter $v$ corresponds to the applied pulling speed in 
SMD simulations). Consequently, $v$ is the product of $\chi \cdot x_{b}$. This leads to the following equation for the bond breaking speed:

$v=\omega_{0} \cdot x_{b} \cdot \exp \left(-\frac{\left(E_{b}-f \cdot x_{b}\right)}{k_{b} \cdot T}\right)$.

This equation can be rewritten in the following way:

$v=v_{0} \cdot \exp \left(\frac{f \cdot x_{b}}{k_{b} \cdot T}\right)$,

with $v_{0}$ as the as the natural bond breaking speed (when no load is applied), defined as:

$v_{0}=\omega_{0} \cdot x_{b} \cdot \exp \left(-\frac{E_{b}}{k_{b} \cdot T}\right)$.

This modified framework enables us to study the dependence between the unfolding force and the bond breaking speed or to calculate the average force at which a bond breaks, at a certain pulling rate. We can rewrite equation (3) in the following way:

$$
f(v)=\frac{k_{b} \cdot T}{x_{b}} \cdot \ln v-\frac{k_{b} \cdot T}{x_{b}} \cdot \ln v_{0}=a \cdot \ln v+b,
$$

where $a=k_{b} \cdot T / x_{b}$ and $b=-k_{b} \cdot T / x_{b} \cdot \ln v_{0}$. Equation (5) predicts that the unfolding force depends logarithmically on the pulling speed in a non-equilibrated system. We note that it contains two parameters $a$ and $b$, which can be calculated exactly from the parameters $x_{b}$ and $E_{b}$ for a certain temperature. 
Equation (5) provides an immediate link between the energy landscape, the pulling rate and the pulling force that is necessary to lower the energy barrier in such a way that the bond can be broken with the applied velocity (Ackbarow, Chen et al. 2007; Ackbarow and Buehler, 2007). Increasing the pulling rate means increasing the off rate and thus the probability of bond rupture. This is only possible through lowering of the energy barrier at the transition point, resulting in a higher bond breaking force $f$ (Ackbarow, Chen et al. 2007; Ackbarow and Buehler, 2007). Further details regarding this model can be found elsewhere (Ackbarow, Chen et al. 2007; Ackbarow and Buehler, 2007).

It is noted that in general, the distance at which a molecule unfolds (parameter $x_{b}$ ) depends on the applied load. However, for a smaller range of pulling speeds, the variation of the distance at which the molecule unfolds depends not or only minutely on the applied speed and can be approximated by the model discussed above. In particular, this behaviour has been confirmed for the particular structure considered in this paper, for the range of pulling speeds of interest (please see (Ackbarow, Chen et al. 2007)).

\section{The nanomechanics of a IF dimer}

Earlier theoretical nanomechanical studies involving atomistic simulation and theoretical analyses have been focused on the mechanics of the IF CC dimer structure (Buehler and Ackbarow 2007; Ackbarow and Buehler, 2007). Here we briefly review these earlier simulation results.

Figure 6 depicts two representative force-strain curves of stretching a CC structure, obtained from molecular dynamics (MD) simulations that show the stretching mechanics of the re vimentin $\mathrm{CC}$ structure, revealing a strong dependence of the mechanical 
properties on the deformation velocity. The MD simulation results have been obtained by studying a vimentin dimer structure in explicit TIP3 water, modeled using the CHARMM force field in NAMD (MacKerell, Bashford et al. 1998; Mackerell 2004). The load has been applied using the Steered Molecular Dynamics (SMD) method (Ackbarow and Buehler, 2007). This approach enables one to control the deformation speed while measuring the force-extension history as the molecule undergoes deformation (for details regarding the analysis of the SMD simulations, please see the original reference (Ackbarow and Buehler, 2007); all simulations have been carried out in explicit water solvent).

A critical measure in these curves is the critical unfolding force $f_{\mathrm{AP}}$, denoted as the angular point (AP). In particular, the MD results show a strong rate dependence of the unfolding mechanism of a dimer, with the existence of two distinct deformation mechanisms, leading to two discrete slopes in the $f-\log (v)$ plot (in agreement with the prediction by equation (5)). This plot is shown in Figure 7. The combination of theoretical analysis and MD simulation enabled to cover many orders of magnitudes of deformation velocity, reaching down to physiological and experimental deformation rates of $1 \mathrm{E}-8 \mathrm{~m} / \mathrm{sec}$ (Ackbarow, Chen et al. 2007; Ackbarow and Buehler, 2007).

The analysis of the atomistic mechanisms of unfolding revealed that at large deformation rates, rupture of single $\mathrm{H}$-bonds at large strain rates controls the unfolding process (steeper slope in Figure 7) (Ackbarow, Chen et al. 2007; Ackbarow and Buehler, 2007). At smaller deformation rates, simultaneous rupture of three H-bonds is the dominating deformation mechanism (less steep slope in Figure 7). The change for this particular CC structure occurs at a pulling velocity of approximately $0.161 \mathrm{~m} / \mathrm{sec}$ (Ackbarow and 
Buehler, 2007). The change in deformation mechanism is reflect in a change of the parameters $E_{b}$ and $x_{b}$ (details can be found in (Ackbarow, Chen et al. 2007; Ackbarow and Buehler, 2007)). The values for $E_{b}$ and $x_{b}$ are summarized in Table 2. The two slopes in Figure 7 correspond to two distinct unfolding mechanisms in the alpha-helical protein structure. This mechanism was directly observed in large-scale MD simulations (Ackbarow, Chen et al. 2007). The transition of the mechanism enables a direct extrapolation to experimental deformation rates by assuming that the observed unfolding mechanism is identical at those pulling rates.

Most importantly for the present analysis, the unfolding force in physiological conditions varies between roughly $50 \mathrm{pN}$ and $200 \mathrm{pN}$ for deformation speeds varying from $5 \mathrm{E}-8$ to $1 \mathrm{E}-6 \mathrm{~m} / \mathrm{sec}$. These unfolding forces are somewhat in agreement with experimental results (Schwaiger, Sattler et al. 2002; Kiss, Karsai et al. 2006) that led to unfolding forces ranging from $25 \mathrm{pN}$ to $110 \mathrm{pN}$ in a range of pulling velocities between $8 \mathrm{E}-8 \mathrm{~m} / \mathrm{sec}$ and $5 \mathrm{E}-7 \mathrm{~m} / \mathrm{sec}$. We emphasize that these experimental studies were carried out on different types of IFs, so that a quantitative comparison is not possible. However, both experiments and theory predict unfolding forces in the range of around slightly below $100 \mathrm{pN}$ to lower hundreds of $\mathrm{pN}$ at physiologically relevant deformation speeds.

In particular, $F_{\mathrm{AP}}=90 \mathrm{pN}$ for a deformation speed of $1 \times 10^{-7} \mathrm{~m} / \mathrm{sec}$, which serves as an example for the subsequent analysis.

\section{Results}


In this paper, we extend earlier work that had been focused on the vimentin dimer and emphasize on the mechanical properties of the next hierarchical assembly level, the tetramer (see Figures 3 and 4). This analysis is a first, simple approach to study the deformation mechanics of these complex protein structures.

Under tensile loading of a vimentin tetramer, the forces are distributed predominantly as tensile load carried by individual CCs, and as shear forces between different CC dimers (see Figure 4). The key to arrive at insight into this question is therefore to consider the interplay of two competing mechanisms:

- Molecular unfolding of the $\mathrm{CC}$ dimer, mediated by rupture of H-bonds (as discussed in Section 2.2), and

- Interdimer sliding, mediated by rupture of the head-CC interaction bonds.

The key question that must be quantified is whether or not the head-CC interactions break first, or the CC unfolding begins first. Which of the two has a lower critical force controls the dominant failure mode. Generally, for strong interdimer adhesion, CC unfolding will occur first. For weak adhesion, interdimer sliding dominates. This is a universal behavior that is expected to control many systems in which sliding versus rupture of the building block represent competing mechanisms of deformation.

The first step in this analysis is the determination of the critical adhesion properties of the dimer-interaction that distinguishes these two deformation regimes.

\section{Critical interdimer adhesion strength}


Here we focus on an estimate of the critical interdimer adhesion to induce interdimer sliding rather than CC unfolding. In other words, the question is how weak should the interdimer interaction be, in order to allow for sliding rather than unfolding, and vice versa?

A first simple model provides some insight into the nanomechanics of this problem. We assume that the shear force transmitted between two $\mathrm{CC}$ dimers is directly proportional to the contact length $L_{C}$ (note that $L_{C}$ corresponds to the total contact length, accounting for the fact that there are two contact regions as shown in Figures 3 and 4; the two contact regions are a consequence of the particular structure of the tetramer with the two head-tail domain adhesion zones),

$F_{\text {tens }}=\tau_{\text {shear }} L_{C}$,

where $F_{\text {tens }}$ is the applied force in the axial molecular direction. This equation can be rewritten to solve for the critical adhesion strength $\tau_{\text {shear,crit }}$ in order to induce unfolding of the CC structure before interdimer sliding occurs:

$$
\tau_{\text {shear,crit }}=\frac{F_{\mathrm{AP}}}{L_{C}},
$$

where $F_{\mathrm{AP}}$ corresponds to the critical unfolding force of each dimer. The analysis reviewed in Section 2.2 provides estimates for the unfolding force of a dimer. For instance, $F_{\mathrm{AP}}=90 \mathrm{pN}$ for a deformation speed of $1 \mathrm{E}-7 \mathrm{~m} / \mathrm{sec}$ (see discussion in Section 2.2). Assuming the contact length $L_{C}=20 \mathrm{~nm}$ (counting the two head-CC interactions, 
each of which is $10 \mathrm{~nm}$ long as illustrated in experiment (Kreplak, Aebi et al. 2004)), the critical shear resistance is $\tau_{\text {shear,crit }}=0.45 \mathrm{pN} / \AA$.

This result suggests that for $\tau_{\text {shear }}>\tau_{\text {shear,crit }}$, unfolding of the $\mathrm{CC}$ structure rather than interdimer sliding would occur. In contrast, for $\tau_{\text {shear }} \leq \tau_{\text {shear,crit }}$, interdimer sliding will be the predominant deformation mode.

In comparison, the intermolecular adhesion of tropocollagen (TC) molecules, mediated by a combination of electrostatic and H-bond interactions, was determined to be $\tau_{\text {shear,TC }}$ $=5.5 \mathrm{pN} / \AA$ by a series of full atomistic MD simulations (Buehler 2006; Buehler 2006). While it is rather difficult to relate this result to the specific geometry present in the head$\mathrm{CC}$ interaction, this value suggests that the critical interdimer adhesion strength could be close to the interactions created by electrostatic and H-bond interactions as in the case of TC.

While the adhesion strength provides a useful measure to obtain an overall estimate of the interdimer adhesion, more details of the chemical interactions must be considered in order to arrive at a more quantitative understanding.

Experimental analyses (Kreplak, Aebi et al. 2004) suggest that the head-CC interaction is dominated by Coulomb 'bonds' or electrostatic interactions, formed by the interaction of partially charged Arg (positive, in the head) and Glu and Asp residues (negative, in the $\mathrm{CC})$. These results suggest that the interaction between the head and $\mathrm{CC}$ domain is formed by formation of a cluster of interdimer weak bonds. In light of these interdimer bonds, it is useful to approximate the critical interdimer adhesion in terms of the critical 
shear force that must be transmitted in each cluster of bonds. The critical force exerted at each interdimer traction site is $F_{\text {shear,crit }}=45 \mathrm{pN}$ (assuming that each adhesion region contributes the same to the shear traction, see also Figure 3). In the following sections, we will consider a statistical model to predict the shear strength for different bond breaking scenarios.

\section{Estimate of deformation mode}

The elucidation of the specific, quantitative interdimer adhesion is a very difficult endeavor, which could for instance be undertaken by carrying out extensive reactive MD simulations that explicitly considers the atomistic geometry and charge transfer.

Here we limit the consideration to a simple theoretical analysis that does not explicitly consider the particular atomistic geometry of the IF structure, but aims at a fundamental investigation of the force levels and rupture mechanisms. The focal point of the analysis is the consideration of two distinct bond breaking mechanism at the head-CC interface. We consider two cases, (i) sequential tensile breaking of interdimer bonds as shown in Figure 8(a), and case (ii), concurrent breaking of interdimer bonds due to homogeneous shear, as shown in Figure 8(b). The reason why these two cases are considered is that the precise mechanism of loading of the interdimer shear interactions remains unclear.

Both cases are treated by considering a statistical model based on the Extended Bell Theory (see Section 2.1), by considering a discrete assembly of weak interaction bonds as shown in Figures 3 and 4. It is assumed that the equilibrium spacing of the interdimer bonds is $r_{0} \approx 3 \AA$, which is the characteristic equilibrium distance for a non-covalent 
bond in organic systems (Sheu, Yang et al. 2003). We assume that the transition state for bond rupture occurs at a critical bond separation of $x_{b}^{*}=1 \AA$ (that is, at a total bond length of $r_{0}+x_{b}^{*}=4 \AA$ ). These values can be deduced from experimental analyses of weak bonding (Sheu, Yang et al. 2003). The two cases are different in how the internal bond breaking mechanisms depends on the laterally applied load, and how the energy barrier changes for different mechanisms.

\section{Sequential bond breaking}

Under sequential bond breaking (Figure 8(a)), the critical breaking force is given by

$f(v)=\frac{k_{b} \cdot T}{x_{b}} \cdot \ln v-\frac{k_{b} \cdot T}{x_{b}} \cdot \ln v_{0}=a \cdot \ln v+b$.

with

$v_{0}=\omega_{0} \cdot x_{b} \cdot \exp \left(-\frac{E_{b}}{k_{b} \cdot T}\right)$

as pointed out above (equations (8) and (9) resemble equations (4) and (5) but are repeated here for convenience). It is noted that $x_{b}$ relates to the global displacement necessary to achieve a local displacement of $x_{b}^{*}$. Therefore, the parameter $x_{b}=1 \AA$ for the case of sequential breaking, since the force is applied directly into the loading direction. 
These equations can now be used to estimate the critical energy barrier to provide sufficient resistance so that the interdimer interactions is stronger than the CC itself. For a deformation speed of $1 \times 10^{-7} \mathrm{~m} / \mathrm{sec}$, the critical energy barrier is approximately 15 $\mathrm{kcal} / \mathrm{mol}$. This estimate is obtained by assuming that the local stretching speed sensed at the head-CC interface is the same as the laterally applied deformation speed. The particular energy barrier is estimated based on the requirement that the adhesion is strong enough to induce unfolding of the $\mathrm{CC}$ structure since the intermolecular bonds are sufficiently strong. In other words, if each interdimer bond has an energy barrier of 15 $\mathrm{kcal} / \mathrm{mol}$ or larger, the interdimer adhesion is stronger than the $\mathrm{CC}$ structure, so that unfolding of the $\mathrm{CC}$ structure is the predominant mechanism (Ackbarow and Buehler, 2007).

It is noted that the energy barrier of $15 \mathrm{kcal} / \mathrm{mol}$ corresponds to the strength of several $\mathrm{H}$ bonds breaking simultaneously, as observed in direct atomistic simulation with explicit water (Ackbarow and Buehler, 2007).

\section{Concurrent bond breaking}

The case of concurrent bond breaking (Figure $8(b)$ ) can be treated similarly as the previous case. With $v_{0, N}$ as the as the natural bond breaking speed of a collection of $N$ bonds (each of which has an energy barrier $E_{b}$ ) defined as

$$
v_{0, N}=\omega_{0} \cdot x_{b} \cdot \exp \left(-\frac{N \cdot E_{b}}{k_{b} \cdot T}\right)
$$


the rupture force at a given deformation speed $v$ is given by

$$
f(v, N)=\frac{k_{b} \cdot T}{x_{b}} \cdot \ln v-\frac{k_{b} \cdot T}{x_{b}} \cdot \ln v_{0, N}=a \cdot \ln v+b
$$

or by considering the expression for the natural bound breaking speed $v_{0, N}$, as

$$
f(v, N)=\frac{k_{b} \cdot T}{x_{b}} \cdot \ln v-\frac{k_{b} \cdot T}{x_{b}} \cdot \ln \left[\omega_{0} \cdot x_{b} \cdot \exp \left(-\frac{N \cdot E_{b}}{k_{b} \cdot T}\right)\right]=a \cdot \ln v+b
$$

The underlying assumption in this model is that the total barrier against rupture is given by the total number of bond that break simultaneously, $N$, times the energy per bond, $E_{b}$.

Also, the parameter $x_{b}$ is larger than in the previous section, since the load application in shear requires a larger shear displacement in order to reach the local transition state for the individual bond $\left(x_{b}^{*}\right)$. Thus in this case, the parameter $x_{b}=2.65 \AA$ (this value is estimated by assuming that sliding occurs without a change in the interdimer distance, and that the bond must be stretched by $1 \AA$ in order to rupture; this leads to a lateral displacement of $2.65 \AA$ ).

In this case the total energy barrier $E_{b, \text { tot }}=N \cdot E_{b}=17.7 \mathrm{kcal} / \mathrm{mol}$. The energy per bond is given by $E_{b}=E_{b, \text { tot }} / N$.

The number of possible bonds that can be formed between the head and $\mathrm{CC}$ domain can be estimated by considering the details of the head-CC interactions. Experiment suggests that each 'bond' consists of an interaction of an Arg residue in the head domain, 
with either an Asp or a Glu residue in the CC segment (see blow-up in Figure 3). The analysis of the specific sequence of amino acids in the head and CC domain reveals that approximately $11-14 \%$ of all residues are polar, charged residues (Strelkov, Herrmann et al. 2002). There are 80 residues in the head domain, half of which fold back to the CC, and half of which fold onto the other dimer, so that there are approximately four Arg residues in contact with the $\mathrm{CC}$, due to the $11-14 \%$ of all residues participating in the bonding. That is, approximately four interdimer 'bonds' can form between the head domain and the $2 \mathrm{~B}$ segment of the $\mathrm{CC}$ in the overlap region.

For this case, the critical energy barrier for the sequential breaking case is estimated to be $4.425 \mathrm{kcal} / \mathrm{mol}$. This value is on the order of the energy of a single H-bond (Sheu, Yang et al. 2003). It is observed that the shear deformation mode requires a lower energy per bond, since multiple bonds contribute to the shear resistance.

\section{Estimate of the interdimer bond energy}

As indicated above and suggested from experimental analyses (Kreplak, Aebi et al. 2004), the head-CC interaction is dominated by Coulomb 'bonds' or electrostatic interactions, as modeled in many empirical force fields (Mayo, Olafson et al. 1990; MacKerell, Bashford et al. 1998; Mackerell 2004). The energy landscape of electrostatic interactions can be approximated using the Coulomb law,

$$
E\left(r_{0}\right)=\frac{1}{4 \pi \varepsilon_{0}} \cdot \frac{\left|q_{1}\right|\left|q_{2}\right|}{r_{0}^{2}},
$$


where $\varepsilon_{0}=8.85 \mathrm{E}-12 \mathrm{C}^{2} \mathrm{~N}^{-1} \mathrm{~m}^{-2}$ (permittivity of free space), and $\left|q_{i}\right|$ describes the two charges (modeled as point charges). Note that $r_{0}$ refers to the equilibrium spacing of the particular Coulomb 'bond' considered. As discussed above, we assume that $r_{0} \approx 3 \AA$, the characteristic equilibrium distance for a non-covalent bond in organic systems. It is assumed that the interdimer bonds are formed between the side chains of the participating amino acid residues without presence of water molecules between the bond. An overview over the molecular geometries of the participating amino acid residues (Arg, Glu, Asp) is provided in Figure 9.

The charges $\left|q_{i}\right|$ can be determined from an analysis of the atomistic charge distribution of the participating residues. All considerations are carried out at $\mathrm{pH}$ 7. The head domain contains strongly positive charges due to arginine (Arg) residues. Since the Arg side chain has a $\mathrm{pK}_{\mathrm{a}}=12.48$, at $\mathrm{pH} 7$ the side chain is deprotonated and charged positively. According to the charge distribution predicted by the CHARMM force field (MacKerell, Bashford et al. 1998), the resulting partial charge of the end of the side chain is $\left|q_{1}\right|=+0.12 \mathrm{C}$ (see Figure 9).

The $1 \mathrm{~A} / 2 \mathrm{~B}$ segment of the $\mathrm{CC}$ domain contains negative charges due to presence of glutamic acid (Glu) residues with $\mathrm{pK}_{\mathrm{a}}=4.07$, as well as aspartic acid (Asp) residues with $\mathrm{pK}_{\mathrm{a}}=3.86$. In Asp and Glu, the side chain is thus protonated, with a total partial charge of the end of the side chain of $-\left|q_{2}\right|=-0.76 \mathrm{C}$ (MacKerell, Bashford et al. 1998).

This information now enables us to estimatea the bond energy of head-CC interactions. The energy barrier to break such a bond is given by $E_{b}=E\left(r_{0}\right)(\operatorname{since} E(r \rightarrow \infty)=0)$, 
with the estimates for $\left|q_{i}\right|$ as discussed above. The resulting energy stored in each bond is approximately $10 \mathrm{kcal} / \mathrm{mol}$.

It is emphasized that the analysis reported here is simplistic, but it provides one with a first estimate of the interdimer adhesion strength. It is noted that the value reported here is in the same order of magnitude as the bond energy reported for electrostatic dominated ligand-receptor binding in streptavidin-biotin complexes (Yuan, Chen et al. 2000), suggesting that the values are reasonable.

\section{Predominant deformation mode}

The analysis in the previous section reveals the predominant deformation mode depends critically on the details of the head-CC interaction. The analysis reported above led to critical energy barriers under two different deformation modes, being $15 \mathrm{kcal} / \mathrm{mol}$ (sequential bond breaking) and $4.25 \mathrm{kcal} / \mathrm{mol}$ (homogeneous bond breaking under shear).

The analysis of electrostatic interactions led to a quantitative estimate for the bond energy in the range of $10 \mathrm{kcal} / \mathrm{mol}$. This value lies in between the two extreme cases considered.

It is possible that the particular interactions are indeed in the range of the critical adhesion, so that under tensile deformation the 'optimal' shear force is transduced to reach the limiting tensile strength within each CC. In other words, the interdimer 'glue' (formed by the head-CC assembly) and the $\mathrm{CC}$ itself are both close to their strength limit. The present analysis is not able to clearly rule out one mechanism over the other. Since the estimate of the electrostatic bonding is rather crude, additional analysis is required to clarify the details of the interaction. 


\section{Discussion and conclusion}

Vimentin IF dimers play a crucial role in determining the large deformation behavior of eukaryotic cells. In this paper we have reported a simple analysis of the interdimer adhesion of vimentin IFs. Our analysis provides insight into critical adhesion forces and critical adhesion energies that lead to either CC dimer unfolding or interdimer sliding. Such models are crucial to advance the understanding of biological process like mechanosensation, which critically depend on the nanomechanical properties of intermediate filaments.

The simple analysis put forward here suggests that the interdimer adhesion provided by the particular interaction of Arg residues with Glu and Asp residues may be at the borderline between $\mathrm{CC}$ unfolding and interdimer sliding. This suggests a possible balance between the two mechanisms. If this were indeed the case, the system would be structured so that the optimal shear force is transduced to reach the limiting tensile strength within each CC. In other words, the structure may be adapted to make optimal use of the interdimer 'glue' material.

We emphasize that while this conclusion is somewhat speculative at this point, it relates well with earlier observations in the structural analysis of the collagen fibril structure (Buehler 2006). It was found that the critical length scale of tropocollagen molecules may be controlled by the driving force to maximize the tensile forces in each molecule and the maximum shear that can be transmitted between molecules. Future investigation will be required to clarify these issues in more detail. 
The study reported here has several limitations. For instance, the quantification of the interdimer adhesion was achieved using a simplistic model based on Coulomb interactions. Atomistic details of the interdimer bonding remains elusive, and could be addressed by extensive MD simulations with reactive force fields, for instance. Further, the details of the interdimer bond rupture processes are entirely unknown. This could, however, significantly influence whether or not interdimer sliding or CC unfolding is the domination mechanism of deformation.

\section{Outlook: Materials science of protein materials}

The discussion of the deformation mechanics of the vimentin tetramer provides an example of how materials science concepts can be used to address the behavior of biological structures. In this section we provide a broader discussion of this field of materials science of protein materials, in light of traditional areas of research in materials science (Buehler and Ackbarow 2007; Buehler 2007 ). Deformation and fracture properties are intimately linked to the atomic microstructure of the material. Whereas crystalline materials show mechanisms such as dislocation spreading or crack extension, biological feature molecular unfolding or sliding, with a particular significance of rupture of chemical bonds such as hydrogen bonds, covalent cross-links or intermolecular entanglement. Much different mechanisms operate at larger length scales, where the interaction with cells and of cells with one another, different tissue types and the influence of tissue remodeling become more evident. The dominance of specific mechanisms is controlled by geometrical parameters as well as the structural arrangement of the protein elementary building blocks, across many hierarchical scales, from nano to macro (see, for instance Figure 1). 
It is known from other fields in materials science that nano- or microscopic structures control the macroscopic material behavior: For example, grain size reduction or confinement leads to an increase of the strength of crystalline metals (Nieh and Wadsworth 1991; Yip 1998; Blanckenhagen, Gumbsch et al. 2001; Wolf, Yamakov et al. 2003). Deformation maps have been proposed to characterize material properties for engineering applications (Frost and Ashby 1982). Discovering similar insight for biological structures and materials represents and important frontier of research. A particularly challenging question is the elucidation of the significance and role of nanostructures for macroscopic properties, that is, carry out sensitivity analyses that show how small-scale features influence larger scale properties.

A major trait of biological materials is the occurrence of hierarchies and, at the molecular scale, the abundance of weak interactions. The presence of hierarchies in biological materials may be vital to take advantage of molecular and sub-molecular features, often characterized by weak interactions, and multiply their properties so that they become visible at larger scales, in order to provide a link between structural organization and function (Fraser 2007). Utilization of weak interactions makes it possible to produce strong materials at moderate temperatures and thus with limited energy use. An important distinction between traditional and biological materials is the geometrical occurrence of defects. While defects are often distributed randomly over the volume in crystalline materials, biological materials consist of an ordered structure that reaches down to the nano-scale. In many biological materials, defects are placed with atomistic or molecular precision, and may play a major role in the material behavior observed at 
larger scales. These features have been observed in bone, nacre, collagenous tissue or cellular protein networks.

The mechanical properties of biological materials have wide ranging implications for biology. In cells for instance, mechanical sensation is used to transmit signals from the environment to the cell nucleus or to control tissue formation and regeneration (Alberts, Johnson et al. 2002; Engler, Sen et al. 2006). The structural integrity and shape of cells is controlled by the cell's cytoskeleton, which resembles an interplay of complex protein structures and signaling cascades arranged in a hierarchical fashion (Alberts, Johnson et al. 2002; Kasza, Rowat et al. 2007). Bone and collagen, providing structure to our body, or spider silk, used for prey procurement, are examples of materials that have incredible elasticity, strength and robustness unmatched by many man-made materials, mainly attributed to its structural formation with molecular precision (Ramachandran 1955; Currey 2002; Kitano 2002; Kitano 2002; An, Sun et al. 2004; Fratzl, Gupta et al. 2004; Buehler 2006; Buehler 2006; Doyle 2007). The transfer of concepts observed in biology into technological applications and new materials design remains a big challenge, with potential huge payoff. In particular, the combination of nanostructural and hierarchical features into materials developments could lead to significant breakthroughs.

What are the most promising strategies in order to analyze these materials? Perhaps, an integrated approach that uses experiment and simulation concurrently could evolve into a new paradigm of materials research. Experimental techniques have gained unparalleled accuracy in both length- and time scales, as reflected in development and utilization of Atomic Force Microscope (AFM) (Prater, Butt et al. 1990; Smith, Schaffer et al. 1999), 
optical tweezers (Dao, Lim et al. 2003; Sun, Luo et al. 2004) or nanoindentation (Tai, Ulm et al. 2006) to analyze biological materials (Lim, Zhou et al. 2006). At the same time, modeling and simulation have evolved into predictive tools that complement experimental analyses. It is now achievable to start from smallest scales - considering electrons and atoms, to reach all the way up to macroscopic scales of entire tissues (Goddard 2006), by explicitly consideration the characteristic structural features at each scale.

Nanoscience and nanotechnology enable us to make structures at the ultimate scale (self assembly, recombinant DNA, utilization of motor proteins for nano-machines and many others). This will perhaps lead to novel complex structural materials, designed from nano to macro. The theoretical progress in understanding hierarchical biological materials will facilitate to use an extended physical space, through the use of multiple hierarchies, in an efficient and controlled manner, that is, lead to a bottom-up structural design on the submacroscopic scale, instead of trial-and-error approaches. For example, the extended design space might serve as mean to realize new physical realities that are not accessible to a single scale, such as material synthesis at moderate temperatures, or fault tolerant hierarchical assembly pathways (Holland 1995), which enable biological systems to overcome the limitations to particular chemical bonds (soft) and chemical elements (organic) present under natural conditions (Ackbarow and Buehler, to be submitted). The increased understanding of the hierarchical design laws might further enable the development and application of new organic and organic-inorganic multi-featured composites (such as assemblies of carbon nanotubes and proteins or polymer-protein composites (Cui, Li et al. 2007; Hule 2007; Winey 2007)), which will mainly consist of 
chemical elements that appear in our environment in an almost unlimited amount $(\mathrm{C}, \mathrm{H}$, $\mathrm{N}, \mathrm{O}, \mathrm{S}$ ). These materials might consequently help to solve human's energy and resource problems (e.g. fossil resources, iron etc.), and allow us to manufacture nano-materials, which will be produced in the future by techniques like recombinant DNA (Petka, Harden et al. 1998; Langer and Tirrell 2004; Smeenk, Otten et al. 2005) or peptide self-assembly (Mershin, Cook et al. 2005; Zhao and Zhang 2006; Zhao and Zhang 2007), techniques where the borders between materials, structures and machines vanish.

Applications of these new materials and structures are new biomaterials, new polymers, new composites, engineered spider silk, new scaffolding tissues, improved understanding of cell-ECM interactions, cell mechanics, hierarchical structures and self-assembly. In addition to the long-term impact in biology, bioengineering and medicine, this research may eventually contribute to our theoretical understanding of how structural features at different scales interact with one another. In light of the 'extended physical design space' discussed above, this may transform engineering approaches not only for materials applications, but also in manufacturing, transportation or designs of networks.

Even though there are still major challenges ahead of us, this progress is amazing and provides one with infinite possibilities and potentials, transforming materials science as a discipline through increased integration of computational approaches in scientific research.

\section{Acknowledgements}


This research was supported by the Army Research Office (ARO), grant number

W911NF-06-1-0291 (program officer Dr. Bruce LaMattina), the Solomon Buchsbaum

AT\&T Research Fund, as well as a National Science Foundation CAREER Award

(CMMI-0642545, program officer Dr. Jimmy Hsia).

\section{References}

Ackbarow, T. and M. J. Buehler (2007). "Superelasticity, energy dissipation and strain hardening of vimentin coiled-coil intermediate filaments: Atomistic and continuum studies." Journal of Materials Science 42: 8771-8787: DOI $10.1007 / \mathrm{s} 10853-007-1719-2$.

Ackbarow, T. and M. J. Buehler (2007). "Molecular mechanics of stutter defects in vimentin intermediate filaments." Experimental Mechanics.

Ackbarow, T. and M. J. Buehler (2007). "Hierarchical coexistence of universality and diversity controls robustness and multi-functionality in protein materials ". Nature Precedings, DOI http://hdl.nature.com/10101/npre.2007.826.1

Ackbarow, T., X. Chen, et al. (2007). "Hierarchies, multiple energy barriers and robustness govern the fracture mechanics of alpha-helical and beta-sheet protein domains." P. Natl. Acad. Sci. USA 104: 16410-16415

Akkermans, R. L. C. and P. B. Warren (2004). "Multiscale modelling of human hair." Philosophical Transactions of the Royal Society of London Series a-Mathematical Physical and Engineering Sciences 362(1821): 1783-1793.

Alberts, B., A. Johnson, et al. (2002). Molecular Biology of the Cell, Taylor \& Francis.

An, K. N., Y. L. Sun, et al. (2004). "Flexibility of type I collagen and mechanical property of connective tissue." Biorheology 41(3-4): 239-246.

Bell, G. I. (1978). "Models for Specific Adhesion of Cells to Cells." Science 200(4342): 618-627.

Blanckenhagen, B. v., P. Gumbsch, et al. (2001). "Dislocation sources in discrete dislocation simulations of thin film plasticity and the Hall-Petch relation." Modelling Simul. Mater. Sci. Eng. 9: 157-169.

Bornschlogl, T. and M. Rief (2006). "Single molecule unzipping of coiled coils: Sequence resolved stability profiles." Physical Review Letters 96(11).

Brockwell, D. J. (2007). "Force denaturation of proteins - an unfolding story." Current Nanoscience 3(1): 3-15.

Bruck, H. A., J. J. Evans, et al. (2002). "The role of mechanics in biological and biologically inspired materials." Experimental Mechanics 42(4): 361-371.

Buehler, M. J. (2006). "Atomistic and continuum modeling of mechanical properties of collagen: Elasticity, fracture and self-assembly." J. Mater. Res. 21(8): 1947-1961.

Buehler, M. J. (2006). "Nature designs tough collagen: Explaining the nanostructure of collagen fibrils." P. Natl. Acad. Sci. USA 103(33): 12285-12290.

Buehler, M. J. (2007 ). "Nano- and micromechanical properties of hierarchical biological materials and tissues " Journal of Materials Science 42(21): 8765-8770. 
Buehler, M. J. and T. Ackbarow (2007). "Fracture mechanics of protein materials." Materials Today 10(9): 46-58.

Burkhard, P., R. A. Kammerer, et al. (2000). "The coiled-coil trigger site of the rod domain of cortexillin I unveils a distinct network of interhelical and intrahelical salt bridges." Structure with Folding \& Design 8(3): 223-230.

Cieplak, M., T. X. Hoang, et al. (2002). "Thermal folding and mechanical unfolding pathways of protein secondary structures." Proteins-Structure Function and Genetics 49(1): 104-113.

Cui, X. Q., C. M. Li, et al. (2007). "Biocatalytic generation of ppy-enzyme-CNT nanocomposite: From network assembly to film growth." Journal of Physical Chemistry C 111(5): 2025-2031.

Currey, J. D. (2002). Bones: Structure and Mechanics. Princeton, NJ, Princeton University Press.

Dao, M., C. T. Lim, et al. (2003). "Mechanics of the human red blood cell deformed by optical tweezers." Journal Of The Mechanics And Physics Of Solids 51(11-12): 2259-2280.

Day, R. and V. Daggett (2007). "Direct observation of microscopic reversibility in singlemolecule protein folding." Journal of Molecular Biology 366(2): 677-686.

Doyle, J. (2007). "Rules of engagement." Nature 446: 860.

Dudko, O. K., G. Hummer, et al. (2006). "Intrinsic rates and activation free energies from single-molecule pulling experiments." Physical Review Letters 96(10).

Engler, A. J., S. Sen, et al. (2006). "Matrix elasticity directs stem cell lineage specification." Cell 126(4): 677-689.

Evans, E. and K. Ritchie (1997). "Dynamic strength of molecular adhesion bonds." Biophysical Journal 72(4): 1541-1555.

Finke, J. M., P. A. Jennings, et al. (2007). "Equilibrium unfolding of the poly(glutamic acid)(20) helix." Biopolymers 86(3): 193-211.

Forman, J. R. and J. Clarke (2007). "Mechanical unfolding of proteins: insights into biology, structure and folding." Current Opinion in Structural Biology 17(1): 5866.

Fraser, P., Bickmore, W. (2007). "Nuclear organization of the genome and the potential for gene regulation." Nature 447(7143): 413-417.

Fratzl, P., H. S. Gupta, et al. (2004). "Structure and mechanical quality of the collagenmineral nano-composite in bone." Journal Of Materials Chemistry 14(14): 2115 2123.

Frost, H. J. and M. F. Ashby (1982). Deformation-mechanism maps, Pergamon Press, Oxford.

Fudge, D. S., K. H. Gardner, et al. (2003). "The mechanical properties of hydrated intermediate filaments: Insights from hagfish slime threads." Biophysical Journal 85(3): 2015-2027.

Fudge, D. S. and J. M. Gosline (2004). "Molecular design of the alpha-keratin composite: insights from a matrix-free model, hagfish slime threads." Proceedings of the Royal Society of London Series B-Biological Sciences 271(1536): 291-299.

Gilli, P., V. Bertolasi, et al. (2004). "Covalent versus electrostatic nature of the strong hydrogen bond: Discrimination among single, double, and asymmetric single-well hydrogen bonds by variable-temperature X-ray crystallographic methods in beta- 
diketone enol RAHB systems." Journal of the American Chemical Society 126(12): 3845-3855.

Goddard, W. A. (2006). A Perspective of Materials Modeling Handbook of Materials Modeling. S. Yip, Springer.

Hanke, F. and H. J. Kreuzer (2006). "Breaking bonds in the atomic force microscope: Theory and analysis." Physical Review E 74(3).

Helfand, B. T., L. Chang, et al. (2004). "Intermediate filaments are dynamic and motile elements of cellular architecture." Journal of Cell Science 117(2): 133-141.

Herrmann, H. and U. Aebi (2004). "Intermediate filaments: Molecular structure, assembly mechanism, and integration into functionally distinct intracellular scaffolds." Annual Review of Biochemistry 73: 749-789.

Holland, J. H. (1995). Hidden Order - How Adaptation Builds Complexity. Reading, MA, Helix Books.

Hule, R., A., Pochan, D., J., (2007). "Polymer Nanocomposites for Biomedical Application." MRS Bulletin 32(4): 5.

Janmey, P. A., U. Euteneuer, et al. (1991). "Viscoelastic Properties of Vimentin Compared with Other Filamentous Biopolymer Networks." Journal of Cell Biology 113(1): 155-160.

Kasza, K. E., A. C. Rowat, et al. (2007). "The cell as a material." Current Opinion in Cell Biology 19(1): 101-107.

Kiss, B., A. Karsai, et al. (2006). "Nanomechanical properties of desmin intermediate filaments." Journal of Structural Biology 155(2): 327-339.

Kitano, H. (2002). "Computational systems biology." Nature 420(6912): 206-210.

Kitano, H. (2002). "Systems biology: A brief overview." Science 295(5560): 1662-1664.

Kreplak, L., U. Aebi, et al. (2004). "Molecular mechanisms underlying the assembly of intermediate filaments." Experimental Cell Research 301(1): 77-83.

Kreplak, L., H. Bar, et al. (2005). "Exploring the mechanical behavior of single intermediate filaments." Journal of Molecular Biology 354(3): 569-577.

Kreplak, L. and D. Fudge (2007). "Biomechanical properties of intermediate filaments: from tissues to single filaments and back." Bioessays 29(1): 26-35.

Langer, R. and D. A. Tirrell (2004). "Designing materials for biology and medicine." Nature 428(6982): 487-492.

Lim, C. T., E. H. Zhou, et al. (2006). "Experimental techniques for single cell and single molecule biomechanics." Materials Science \& Engineering C-Biomimetic and Supramolecular Systems 26(8): 1278-1288.

Maccallum, J. L., M. S. Moghaddam, et al. (2007). "Hydrophobic association of \{alpha\}helices, steric dewetting, and enthalpic barriers to protein folding." Proc Natl Acad Sci U S A 104(15): 6206-10.

Mackerell, A. D. (2004). "Empirical force fields for biological macromolecules: Overview and issues." Journal Of Computational Chemistry 25(13): 1584-1604.

MacKerell, A. D., D. Bashford, et al. (1998). "All-atom empirical potential for molecular modeling and dynamics studies of proteins." Journal Of Physical Chemistry B 102(18): 3586-3616.

Mayo, S. L., B. D. Olafson, et al. (1990). "Dreiding - A Generic Force-Field For Molecular Simulations." Journal Of Physical Chemistry 94(26): 8897-8909. 
Mershin, A., B. Cook, et al. (2005). "A classic assembly of nanobiomaterials." Nature Biotechnology 23(11): 1379-1380.

Mitsui, K., K. Nakajima, et al. (2000). "Dynamic measurement of single protein's mechanical properties." Biochemical and Biophysical Research Communications 272(1): 55-63.

Moir, R. D. and T. P. Spann (2001). "The structure and function of nuclear lamins: implications for disease." Cellular and Molecular Life Sciences 58(12-13): 17481757.

Mucke, N., L. Kreplak, et al. (2004). "Assessing the flexibility of intermediate filaments by atomic force microscopy." Journal of Molecular Biology 335(5): 1241-1250.

Mucke, N., T. Wedig, et al. (2004). "Molecular and biophysical characterization of assembly-starter units of human vimentin." Journal of Molecular Biology 340(1): 97-114.

Nieh, T. G. and J. Wadsworth (1991). "Hall-Petch relation in nanocrystalline solids." Scripta Met. 25(4).

Omary, M. B., P. A. Coulombe, et al. (2004). "Mechanisms of disease: Intermediate filament proteins and their associated diseases." New England Journal of Medicine 351(20): 2087-2100.

Ortiz, V., S. O. Nielsen, et al. (2005). "Unfolding a Linker between Helical Repeats." Journal of Molecular Biology 349(3): 638-647.

Paramore, S. and G. A. Voth (2006). "Examining the influence of linkers and tertiary structure in the forced unfolding of multiple-repeat spectrin molecules." Biophysical Journal 91(9): 3436-3445.

Petka, W. A., J. L. Harden, et al. (1998). "Reversible hydrogels from self-assembling artificial proteins." Science 281(5375): 389-392.

Prater, C. B., H. J. Butt, et al. (1990). "ATOMIC FORCE MICROSCOPY." Nature 345(6278): 839-840.

Ramachandran, G. N., Kartha, G. (1955). "Structure of collagen." Nature 176: 593-595.

Randles, L. G., R. W. S. Rounsevell, et al. (2007). "Spectrin domains lose cooperativity in forced unfolding." Biophysical Journal 92(2): 571-577.

Rohs, R., C. Etchebest, et al. (1999). "Unraveling proteins: A molecular mechanics study." Biophysical Journal 76(5): 2760-2768.

Root, D. D., V. K. Yadavalli, et al. (2006). "Coiled-coil nanomechanics and uncoiling and unfolding of the superhelix and alpha-helices of myosin." Biophysical Journal 90(8): 2852-2866.

Rose, A. and I. Meier (2004). "Scaffolds, levers, rods and springs: diverse cellular functions of long coiled-coil proteins." Cellular and Molecular Life Sciences 61(16): 1996-2009.

Schietke, R., D. Brohl, et al. (2006). "Mutations in vimentin disrupt the cytoskeleton in fibroblasts and delay execution of apoptosis." European Journal of Cell Biology 85(1): 1-10.

Schwaiger, I., C. Sattler, et al. (2002). "The myosin coiled-coil is a truly elastic protein structure." Nature Materials 1(4): 232-235.

Sheu, S. Y., D. Y. Yang, et al. (2003). "Energetics of hydrogen bonds in peptides." Proceedings of the National Academy of Sciences of the United States of America 100(22): 12683-12687. 
Smeenk, J. M., M. B. J. Otten, et al. (2005). "Controlled assembly of macromolecular beta-sheet fibrils." Angewandte Chemie-International Edition 44(13): 1968-1971.

Smith, B. L., T. E. Schaffer, et al. (1999). "Molecular mechanistic origin of the toughness of natural adhesives, fibres and composites." Nature 399(6738): 761-763.

Smith, T. A., P. D. Hempstead, et al. (2003). "Modeling alpha-helical coiled-coil interactions: The axial and azimuthal alignment of $1 \mathrm{~B}$ segments from vimentin intermediate filaments." Proteins-Structure Function and Genetics 50(2): 207-212.

Strelkov, S. V., H. Herrmann, et al. (2003). "Molecular architecture of intermediate filaments." Bioessays 25(3): 243-251.

Strelkov, S. V., H. Herrmann, et al. (2002). "Conserved segments 1A and 2B of the intermediate filament dimer: their atomic structures and role in filament assembly." EMBO Journal 21(6): 1255-1266.

Strelkov, S. V., J. Schumacher, et al. (2004). "Crystal structure of the human lamin a coil 2B dimer: Implications for the head-to-tail association of nuclear lamins." Journal of Molecular Biology 343(4): 1067-1080.

Sun, Y. L., Z. P. Luo, et al. (2004). "Stretching type II collagen with optical tweezers." Journal Of Biomechanics 37(11): 1665-1669.

Tai, K., F. J. Ulm, et al. (2006). "Nanogranular origins of the strength of bone." Nano Letters 11: 2520-2525

Toivola, D. M., G. Z. Tao, et al. (2005). "Cellular integrity plus: organelle-related and protein-targeting functions of intermediate filaments." Trends in Cell Biology 15(11): 608-617.

Wang, N. and D. Stamenovic (2002). "Mechanics of vimentin intermediate filaments." Journal of Muscle Research and Cell Motility 23(5-6): 535-540.

Wiita, A. P., S. R. K. Ainavarapu, et al. (2006). "Force-dependent chemical kinetics of disulfide bond reduction observed with single-molecule techniques." Proceedings of the National Academy of Sciences of the United States of America 103(19): $7222-7227$.

Wilson, K. L., M. S. Zastrow, et al. (2001). "Lamins and disease: Insights into nuclear infrastructure." Cell 104(5): 647-650.

Winey, K. I., Vaia R.A., (2007). "Polymer Nanocomposites." MRS Bulletin 32(4): 5.

Wolf, D., V. Yamakov, et al. (2003). "Deformation mechanism and Inverse Hall-Petch behavior in nanocrystalline materials." Z. Metallk. 94: 1052-1061.

Wolgemuth, C. W. and S. X. Sun (2006). "Elasticity of alpha-helical coiled coils." $\underline{\text { Phys }}$ Rev Lett 97(24): 248101.

Yip, S. (1998). "The strongest size." Nature 391: 532-533.

Yuan, C. B., A. Chen, et al. (2000). "Energy landscape of streptavidin-biotin complexes measured by atomic force microscopy." Biochemistry 39(33): 10219-10223.

Zhao, X. J. and S. G. Zhang (2006). "Molecular designer self-assembling peptides." Chemical Society Reviews 35(11): 1105-1110.

Zhao, X. J. and S. G. Zhang (2007). "Designer self-assembling peptide materials." Macromolecular Bioscience 7(1): 13-22. 


\section{Tables and table legends}

\begin{tabular}{|l|l|}
\hline Cytoskeleton & $\begin{array}{l}\text { A composite inside the cell consisting of three different networks: } \\
\text { Actin filaments, microtubules and intermediate filaments. The } \\
\text { intermediate filament network is in the focus of this paper. These } \\
\text { networks connect the nucleus (nuclear membrane) with the plasma } \\
\text { membrane and are furthermore responsible for the organization } \\
\text { inside the cell. }\end{array}$ \\
\hline $\begin{array}{l}\text { Intermediate } \\
\text { filaments (IFs) }\end{array}$ & $\begin{array}{l}\text { One of the three components of the cytoskeleton; mainly responsible } \\
\text { for the large deformation behavior of the cell. }\end{array}$ \\
\hline $\begin{array}{l}\text { Cross bridging } \\
\text { proteins }\end{array}$ & $\begin{array}{l}\text { Cross bridging proteins form connections inside each cytoskeletal } \\
\text { network as well as connections between different networks. }\end{array}$ \\
\hline Dimer & $\begin{array}{l}\text { A dimer is the elementary building block of an IF fiber. This protein } \\
\text { consists of a head domain, a tail domain and an extremely elongated } \\
\text { coiled-coil rod. A coiled-coil is a superhelix that consists of two alpha } \\
\text { helices that twist around each other. }\end{array}$ \\
\hline Assembly & $\begin{array}{l}\text { Individual IF dimers assemble systematically and hierarchically into } \\
\text { filaments (Figure 1 (b)). Two dimers build a tetramer, two tetramers } \\
\text { build an octamer and four octamers build a unit length filament (ULF). } \\
\text { Once this level of assembly is reached, ULFs ally longitudinally into } \\
\text { long fibers. }\end{array}$ \\
\hline Residue & $\begin{array}{l}\text { The primary structure of a protein consists of a sequence of amino } \\
\text { acids. One residue is thus one amino acid in the polypeptide } \\
\text { backbone. }\end{array}$ \\
\hline
\end{tabular}

Table 1: Summary of different biological terms and concepts used in this paper (adapted from (Ackbarow and Buehler, 2007)). 


\begin{tabular}{|l|c|c|}
\hline Deformation regime & $\begin{array}{c}\text { Energy barrier } E_{b} \\
\text { (kcal/mol) }\end{array}$ & $\begin{array}{c}\text { Distance } x_{b} \\
\text { (Angstrom) }\end{array}$ \\
\hline Slow & 15.00 & 1.00 \\
\hline Fast & 5.60 & 0.17 \\
\hline
\end{tabular}

Table 2: Estimate of the energy landscape for the two deformation regimes under slow and fast deformation (Ackbarow and Buehler, 2007). 


\section{Figures and figure captions}

Figure 1: Hierarchical structure of the IF network in cells. Through carefully following the various steps of assembly (Smith, Hempstead et al. 2003; Kreplak, Aebi et al. 2004) it was shown that dimers associate to fibrils, which form the second level of the hierarchy. In vivo, these fibrils can reach a length of up to several $\mu \mathrm{m}$ and consist of 16 dimers in cross-section. The third level of hierarchy consists of three-dimensional IF-networks inside the cell, reinforcing the plasma membrane (Herrmann and Aebi 2004; Mucke, Kreplak et al. 2004; Strelkov, Schumacher et al. 2004). Inside the network, IF associated proteins such as plectins generate the connection between individual IFs as well as between other cytoskeletal components. The characteristic loading condition of full length filaments is tensile loading. Due to this tensile load, each dimer is subject to a tensile load if the cell undergoes large deformation. Tensile load at the tetramer and higher level is carried through shear load between individual dimers. Figure 2: Geometry of the dimeric building block in vimentin. The dimers, approximately $45 \mathrm{~nm}$ long, are the elementary building blocks of vimentin intermediate filaments. A dimer consists of a head, tail and an elongated rod domain which is divided into four alphahelical coiled coils (1A, 1B, 2A, 2B) connected through linkers (plotted in green) (Strelkov, Herrmann et al. 2003). The head and tail domains (indicated by "heads" and "tails" in the figure) are crucial for assembly of dimers into tetramers and larger scale structures.

Figure 3: Schematic of the tetramer, formed by interaction of two dimers. Two dimers are assembled in the $A_{11}$ assembly pattern antiparallel, approximately half-staggered, as suggested in experimental studies (Kreplak, Aebi et al. 2004). Interdimer adhesion is 
facilitated by the interaction of the head domain with the other dimer (indicated with the red color). The head domain is primarily positively charged (approximately 15\% Arg residues), and folds back into the $\mathrm{CC} 1 \mathrm{~A}$ domain, negatively charged, and also connects to the $2 \mathrm{~B}$ domain of the other dimer, which is also negatively charged (the $1 \mathrm{~A}$ and $2 \mathrm{~B}$ domains contain approximately $11 \%$ Glu and Asp residues). The details of the interdimer bonding is schematically shown in the blow-up (lower part of the figure).

Figure 4: Schematic representation of the dimer geometry and the interdimer traction. Subplot (a) depicts the dimer structure under lateral tensile loading. Subplot (b) depicts the upper half of the structure, showing the internal tractions, transmitted via the head-CC interaction as illustrated in the blow-up in Figure 3.

Figure 5: The graph depicts the energy as a function of deformation along a deformation variable (upper plot), along a particular pathway that leads to bond rupture; where $F$ is the applied force, and $x_{b}$ is the displacement in the direction of the applied force. In the schematic below, three HBs (indicated by the red color) break simultaneously. Thus, $x_{b}$ corresponds to the lateral displacement that is necessary to overcome the bond breaking distance of a $\mathrm{HB}$, in the particular atomistic geometry present in the $\mathrm{CC}$, and $E_{b}$ corresponds to the energy barrier of $3 \mathrm{H}$-bonds.

Figure 6: Force-strain curves for mechanical tensile deformation of a $\mathrm{CC}$ vimentin dimer, as reported in an earlier study (Ackbarow and Buehler in press). The continuous curves represent results obtained from a theoretical model based on the Bell Theory that enables one to predict the unfolding mechanics for a wide range of pulling velocities. The first regime (I) represents elastic deformation, up to approximately $10 \%$ to $25 \%$ 
tensile strain. This regime is followed by a plateau region (regime (II)) during which unfolding of the $\mathrm{CC}$ structure occurs, at approximately constant force level. The last regime (III) displays significant strain hardening, during which the coiled super-helix is lost and the protein backbone is being stretched, leading to a significant increase in stiffness. The angular point is indicated with the ' $x$ ' symbol.

Figure 7: Dimer unfolding force, and interdimer sliding force as a function of pulling velocity, as reported in (Ackbarow and Buehler in press). The plot contains results of MD simulations, experimental results as well as the prediction based on the Extended Bell Theory. All simulation results display a logarithmic dependence of the unfolding force on the pulling velocity, in agreement with the predictions by the Extended Bell Theory. The change of mechanism at approximately $0.161 \mathrm{~m} / \mathrm{sec}$ pulling velocity is due to a change in the rupture mechanism of $\mathrm{H}$-bonds.

Figure 8: Schematic to illustrate possible interdimer bond breaking mechanism. Subplot (a) depicts sequential breaking, which would occur if the load is applied perpendicular to the molecule axis. Subplot (b) depicts concurrent bond breaking, which would occur if shear load in the direction of the molecular axis is applied.

Figure 9: Interdimer interaction, atomistic details. Interdimer interaction is facilitated by electrostatic interactions between the head and $2 \mathrm{~A}$ domain of the $\mathrm{CC}$ structure. Based on sequence analyses of the vimentin structure, it has been confirmed that the head domain is rich in Arg amino acids (partially positive charged), whereas the $2 \mathrm{~A}$ domain of the $\mathrm{CC}$ is rich in Asp and Glu amino acids, which are partially negative charged at $\mathrm{pH} 7$. 
Eukaryotic cell

Cytoskeleton structure

Full length filaments

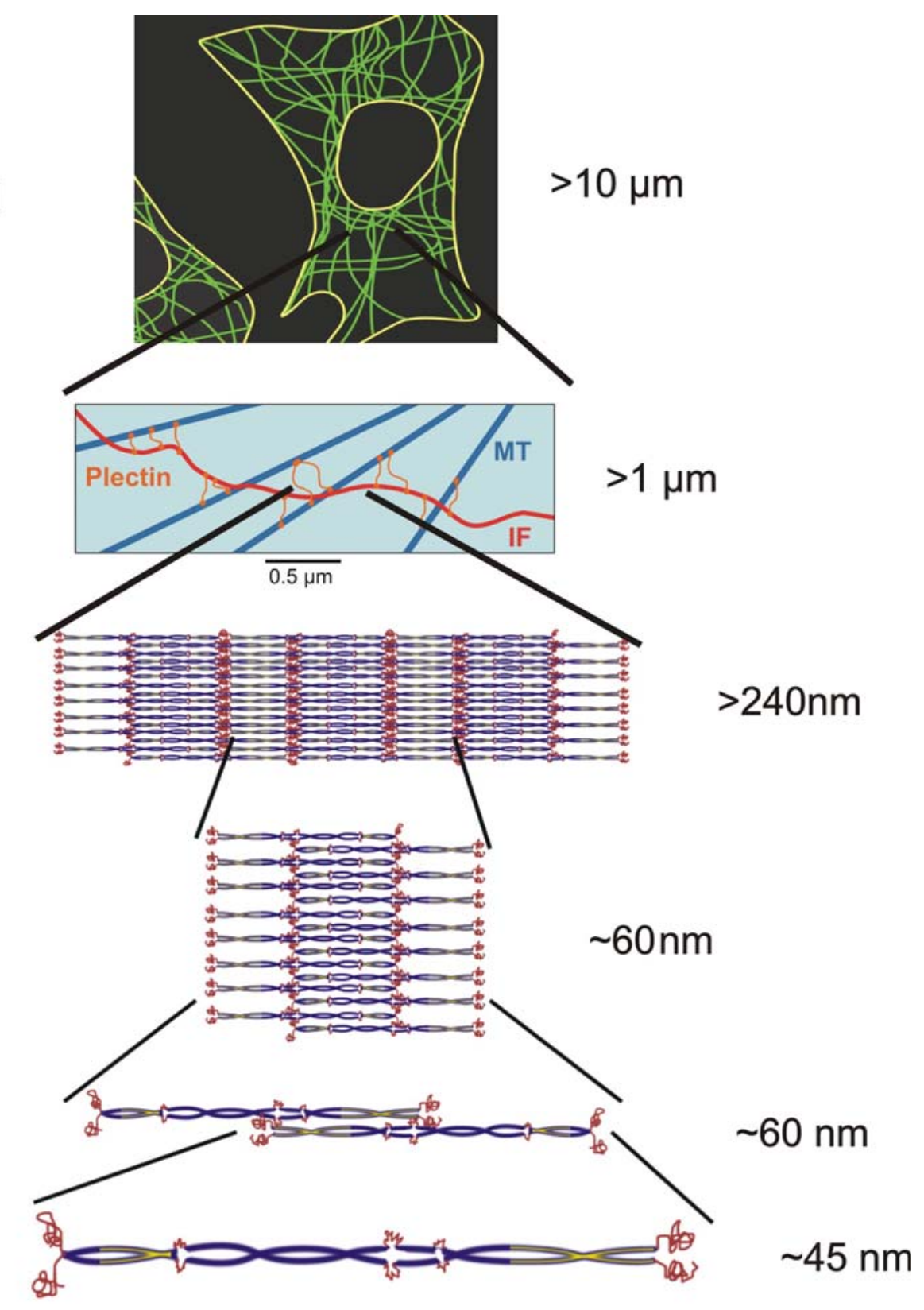

Tetramers

(2 dimers)

Dimer

(elementary

buildingblocks)

Unitlength

(8 tetramers)

Figure 1 


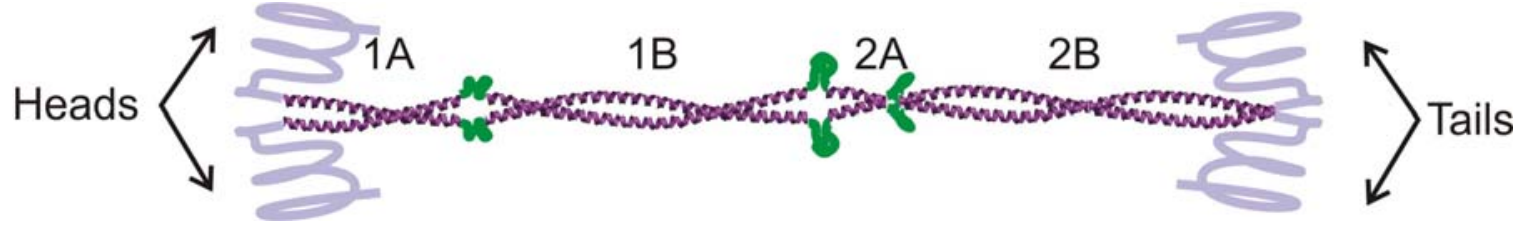

Figure 2 


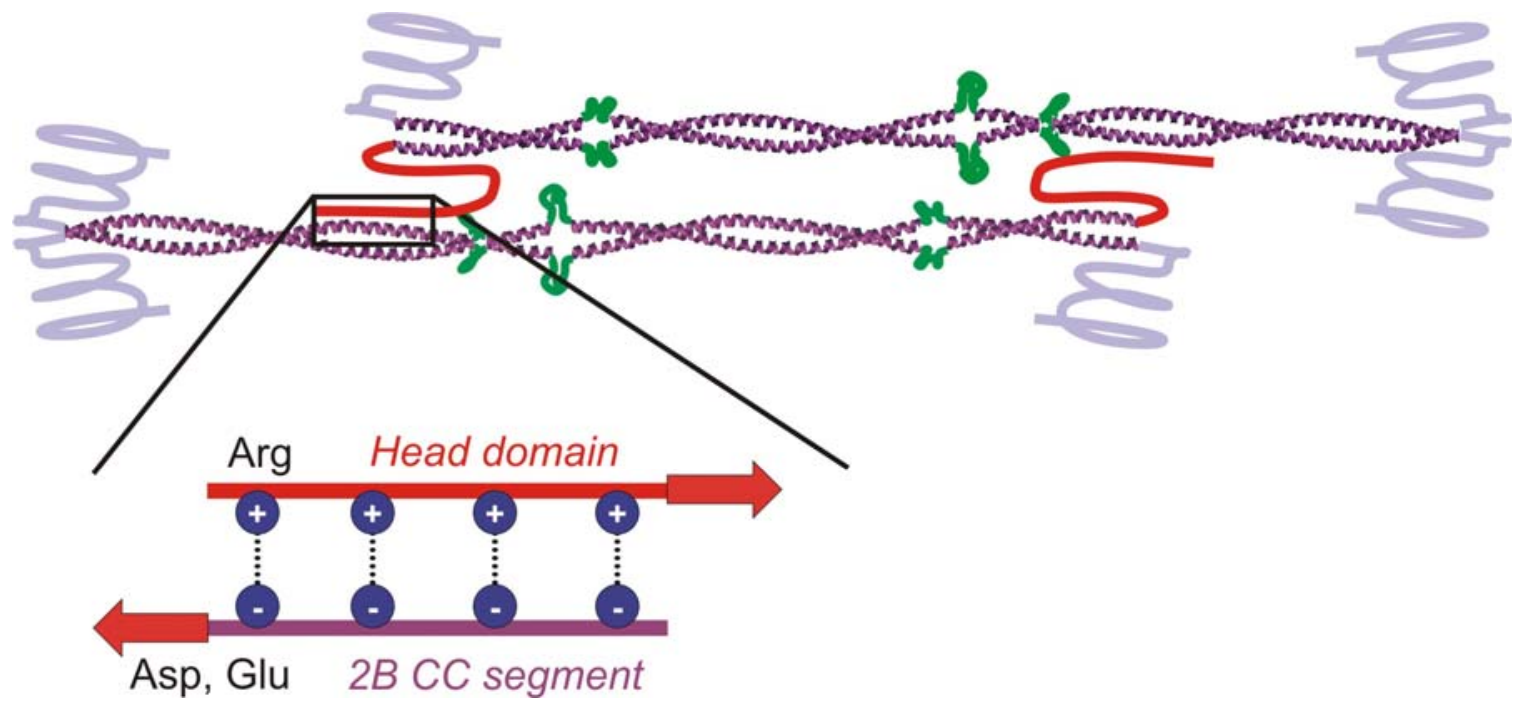

Figure 3 
(a)

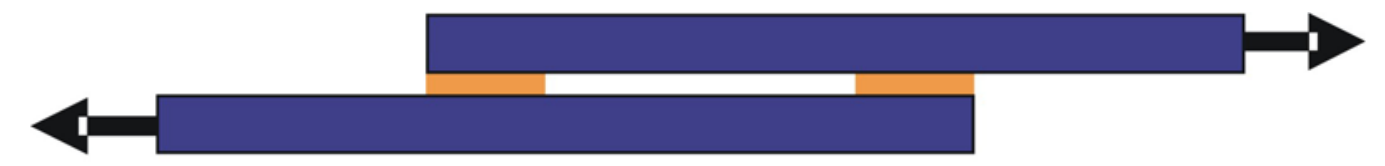

(b)

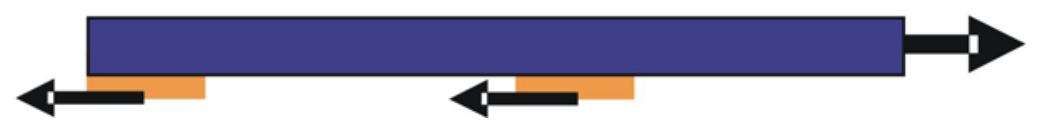

Figure 4 

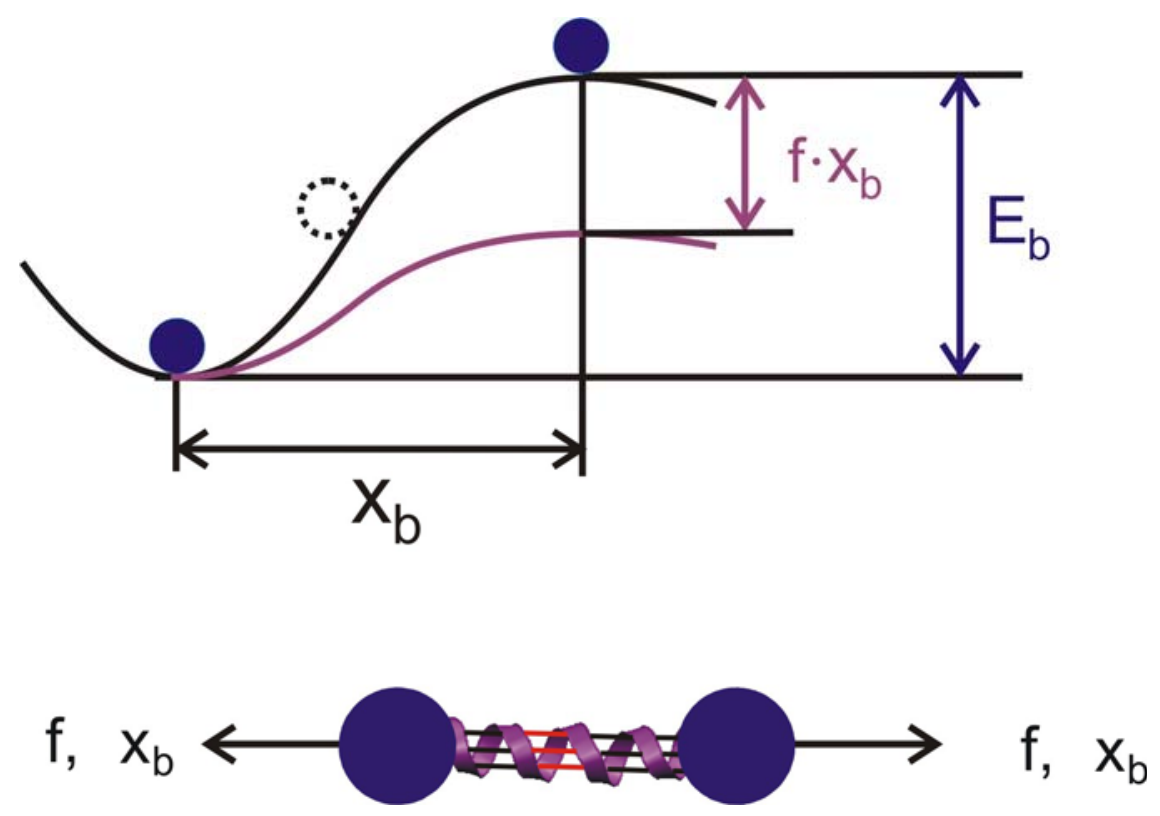

Figure 5 


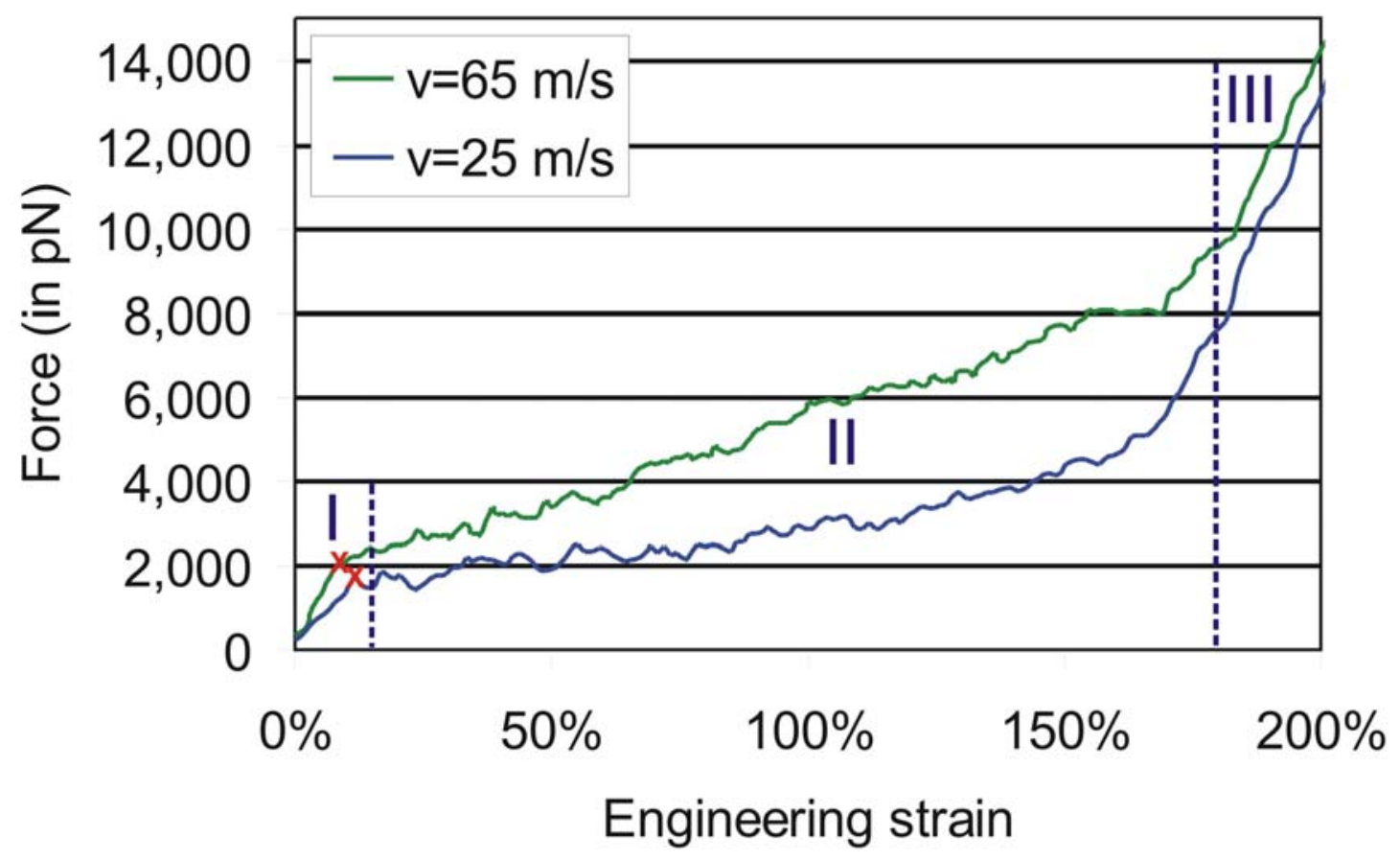

Figure 6 


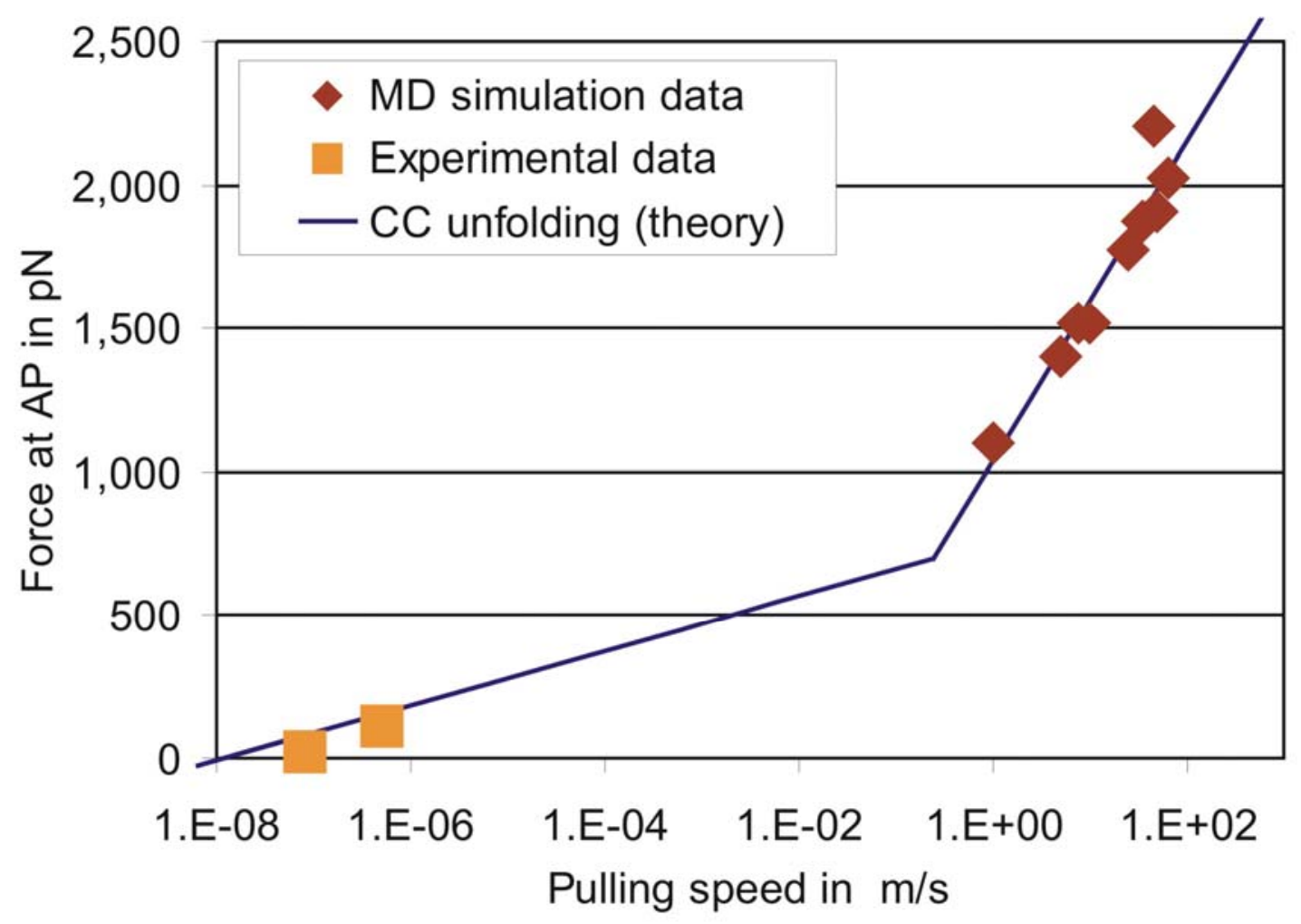

Figure 7 
(a)

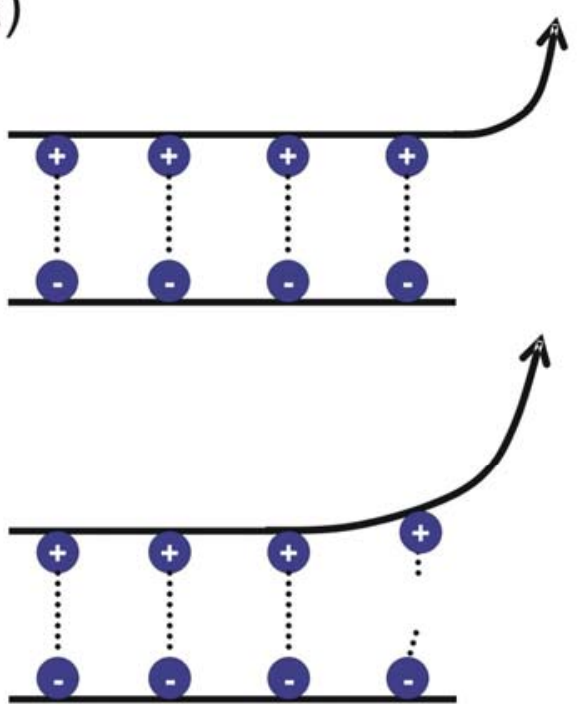

(b)
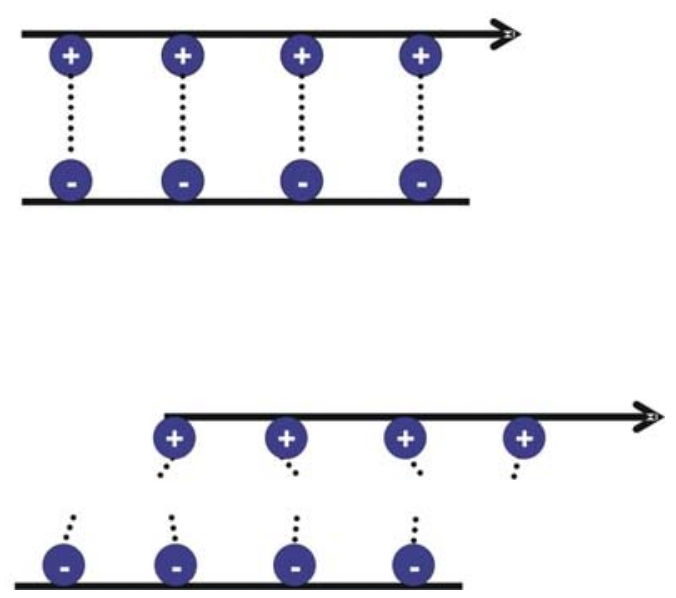

Figure 8 


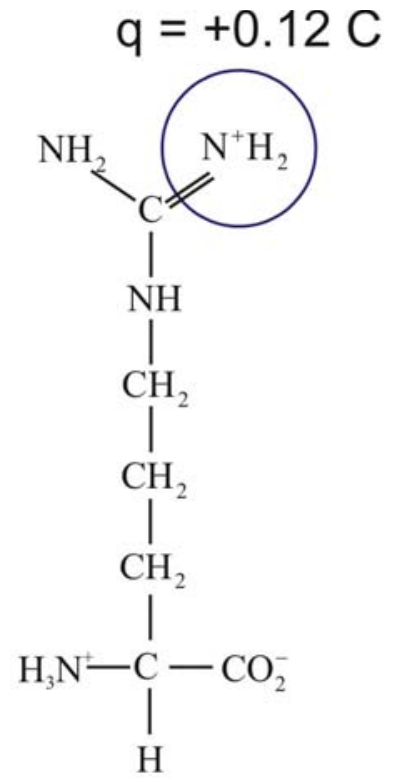

Arginine (Arg)

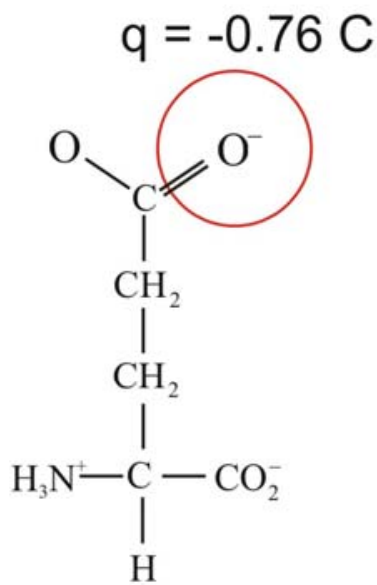

Glutamic acid (Glu)

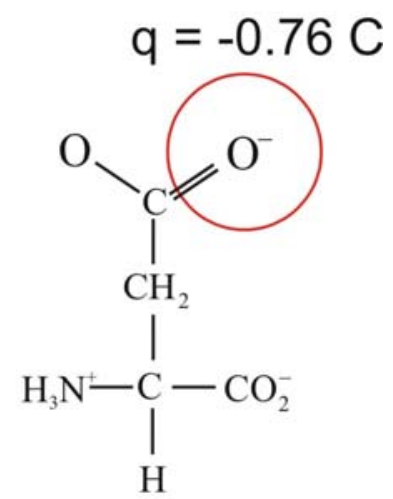

Aspartic acid (Asp)

Figure 9 